\title{
How can we study cardiopulmonary resuscitation and cardiac arrest in animals: a review
}

\begin{abstract}
Animal research is essential to propose new therapeutic approaches limiting the sequels of cardiac arrest in human and veterinary medicine. The ultimate goal is to improve the long term prognosis and neurological recovery. For such investigations, the use of animal models seems unavoidable as cardiac arrest provokes multiple visceral consequences as well as inflammatory response and coagulopathy. These models allow not only a better understanding of the pathophysiology but also the investigation of new treatment strategies to improve basic life support efficiency or prevent multi-organ failure. In this review, we are summarizing the characteristics of the main animal models used in resuscitation sciences. The choice of the species and methods of evaluation of cardiac arrest is crucial. These studies often concern human medicine but they can also address fundamental questions for veterinary research as they are mostly based on animal research.
\end{abstract}

Keywords: cardiac arrest, animal research, resuscitation
Volume 3 Issue 2 - 2016

\author{
Blondel M,',2 Kohlhauer M,' Zilberstein L,' \\ Tissier $\mathbf{R}^{\prime}$ \\ 'Ecole Nationale Vétérinaire d'Alfort, Maisons-Alfort, France \\ ${ }^{2}$ Ecole Nationale Vétérinaire de Toulouse, Toulouse, France
}

Correspondence: Renaud Tissier, Ecole Nationale Vétérinaire d'Alfort, 7 avenue du Général de Gaulle, 94704 Maisons-Alfort cedex, France, Tel +3366047 944I, Email rtissier@vet-alfort.fr

Received: December 15, 2015 | Published: February 15, 2016
Abbreviations: CPR, cardiopulmonary resuscitation; ROSC, resumption of spontenaeous circulation; VF, ventricular fibrillation

\section{Introduction}

Cardiac arrest is an important public health issue in humans in industrialized countries with, e.g., 35,000 to 40,000 new cases each year in France. ${ }^{1,2}$ Despite increasing implementation of cardiopulmonary resuscitation (CPR) in the field, resumption of spontaneous circulation (ROSC) is typically obtained in less than $35 \%$ of these patients. Resuscitated victims often present a post cardiac arrest syndrome with a complex physiopathology combining hemodynamic failure, neurological dysfunction and multi-visceral injury, leading to an additional worsening of the prognosis. Ultimately, less than $8 \%$ of the patients survive with favorable neurological function at hospital discharge. ${ }^{3-5}$

In this context, experimental research is crucial to provide new therapeutic approaches increasing CPR efficiency and initial resuscitation, as well as long term prognosis and neurological recovery. For such investigations, the use of animal models seems unavoidable as cardiac arrest provokes multiple visceral consequences as well as inflammatory response and coagulopathy. These models allow not only a better understanding of the pathophysiology but also the investigation of new treatment strategies to improve CPR efficiency or prevent multi-organ failure. This field of research can also address fundamental questions for veterinary research, beyond the relevance for human medicine.

\section{Impact of species-differences}

In general, animal models of cardiac arrest are using rodents, rabbits, pigs or dogs. All these species present anatomical or physiological specificities that should been considered in the design of the study, depending upon the specific goals and corresponding end-points.

\section{Cardiac arrest in rodents}

As usual in biomedical research, rodents are widely used in resuscitation sciences due to their relative ease of manipulation, low cost and extensive possibilities of mechanistic investigations with genetically modified animals. Obviously, this use is associated with several limitations related to their cardiovascular anatomy and physiology. On the one hand, rodents present two different cranial venae cavae (right and left) with a coronary sinus formed by the proximal part of the left cranial vena cava. ${ }^{6}$ This directly modifies the coronary perfusion during cardiac massage through different venous return as compared to large animals. ${ }^{7}$ In addition, due to their very low body mass, cardiac massage could make blood circulating through direct heart compression, while it is mostly acting through thoracic pumping in large animals. On the other hand, spontaneous heart rate is much higher in rodents than in large animals and humans as usual values average $260-450$ or $500-600$ beats per minute in awake rats and mice, respectively. ${ }^{7,8}$ This directly impacts the required chest compression rate in those species as mice should undergo $\approx 400$ compressions per min to be resuscitated. ${ }^{9-14}$ Magnetic resonance imaging also showed that usual ejection fraction are higher in mice as compared to humans ( $75 \%$ vs $55 \%) \cdot{ }^{15}$ Due to their very small body size, the ratio between the left ventricle and the body weight is also much higher in mice than in humans.

During cardiac arrest, rodents also present several electrophysiological differences related to their high physiological heart rate. As compared to humans, ionic currents are very specific with high concentration of $\mathrm{K}+$ channels. ${ }^{16}$ As a consequence, rats and mice present very short action potential and refractory period. Subsequently, ventricular fibrillation (VF) is able to convert spontaneously in these species, i.e., within the first 1-2min following VF induction. Experimentally, prolonged electrical stimulation should be delivered to obtain sustained VF in these species. ${ }^{7}$

Therefore, rodents are mostly used for exploratory and mechanistic studies. They can also be used for survival studies and evaluation 
of neurological outcome as learning tests or motor behavior measurements are well established in these species. However, a main limitation remains that mice and rats present lissencephalic brains, which may be quite different from human brain regarding cognitive function or sensibility to ischemia. ${ }^{17}$

\section{Cardiac arrest in rabbits}

In experimental research, rabbits are widely used for cardiovascular, osteoarticular and respiratory studies. They can be a good "intermediate" between rodents and large animals, with easier manipulation, blood sampling and cardiovascular monitoring than rodents, while not requiring complex infrastructure and high costs such as large animals do. Interestingly, the electrical activity of the rabbit heart is believed to be close to that of the human heart regarding ionic currents and action potential. ${ }^{18}$ Left ventricular ejection fraction, volumes and heart to body weight ratio are also very similar to human values. ${ }^{15}$ However, the physiological heart rate is still high as compared to humans or large animals (180-250 beats per minutes). Regarding the neurological function, rabbits are comparable to rodents. They have a lissencephalic brain, making the neurological function quite different from humans ${ }^{17}$ However, neurological evaluation is relatively easy to perform through the use of behavioral or postural neurological tests. ${ }^{19}$ For example, it has been described that postural tests could be actually repeatable and related to the severity of the ischemic insult in a model of stroke injury. ${ }^{20}$ However, these evaluations are less commonly used than for rodents.

\section{Large animal models of cardiac arrest}

Large animal models of cardiac arrest are mostly using pigs (i.e., usually swine) or dogs. The latter are less and less used currently. Both species present very close anatomical and physiological characteristics as compared to humans. ${ }^{21}$ However, some differences exist regarding coronary circulation and cardiac electrophysiology. First, pigs do not have native coronary collateral circulation as compared to dogs and humans. Second, they present important variations in the His bundle or Purkinje fibers anatomy with a subsequently different electrical conduction. ${ }^{22}$ Third, their conduction system contains larger numbers of adrenergic and cholinergic fibers than humans in the atrioventricular node and the left and right bundle branches. ${ }^{23}$ This increases heart excitability and should be considered for electrophysiological studies and evaluation of defibrillation strategies. On the contrary, dogs have very close cardiac electrophysiological properties as compared to humans. ${ }^{24}$ Therefore, they might be an "ideal" model for arrhythmia studies but their wide use raises ethical and economical issues. ${ }^{25}$ Pigs are therefore the most commonly used large animals for cardiac arrest studies. Importantly, reliable neurological tests are also available in these species after cardiac arrest. ${ }^{26-31}$

\section{Different methods for the induction of car- diac arrest in animals}

In the clinical setting, cardiac arrest is classified in two categories according to the initial rhythm, i.e., shockable or non-shockable cardiac arrest, which should be treated or not by electrical shocks, respectively. These two categories could be considered as two different physiopathological entities with distinct animal models..$^{32-34}$

\section{Models of shockable cardiac arrest}

Shockable cardiac arrest is caused by disturbances of electrical flux within the myocardium, leading to ventricular fibrillation or ventricular tachycardia.
Electrical induction of ventricular fibrillation: Ventricular fibrillation can be induced by external transthoracic electric shocks. ${ }^{33}$ This is mostly used in large animals. The current intensity and its timing of application during the cardiac cycle are crucial to efficiently induce VF. For example, if the current is delivered outside the vulnerable period or if the magnitude exceeds the limits of vulnerability, VF cannot be obtained. ${ }^{35}$ In small animals, this method could not be implemented as VF could convert spontaneously after a short stimulus. ${ }^{7}$ Shockable cardiac arrest could also be induced with less powerful current through endocavitary electrodes. For example, an alternating current (1-5mA during 1.5 minutes) efficiently induces VF in pigs when passed in the right ventricular endocardium. ${ }^{36,37}$ In small animals, acupunctures needles could also be placed transcutaneously on the thoracic wall to stimulate the heart. However, this method requires higher current intensity as compared to the direct stimulation of the heart and this could lead to lung injury. An intermediate method consists in the electrical stimulation of the heart through one subcutaneous thoracic electrode and another electrode inserted into the esophagus. This technique has been developed in rodents ${ }^{38,39}$ and rabbits ${ }^{40,41}$ but canal so be used in swine. ${ }^{42}$ For example, a sustained transesophageal alternating current stimulation $(50 \mathrm{~Hz}, 18 \mathrm{~V})$ during 60 seconds has been shown to induce VF in mice. ${ }^{38}$ In rats and rabbits, VF can be induced with a $50-60 \mathrm{~Hz}$ current of $1-5 \mathrm{~mA}$ during 3 minutes. ${ }^{40,43,44}$ In another study, a rapid pacing with three periods of 5 seconds of transesophageal stimulation at $30 \mathrm{~Hz}$ efficiently induces VF in rats. ${ }^{45}$ Thermal injuries are negligible in the esophagus and did not appear to affect the heart. . $^{38,39}$

Myocardial ischemia-induced ventricular fibrillation: Ventricular arrhythmias can also be induced by myocardial ischemia, ${ }^{46}$ which is probably the most clinically relevant strategy. For this purpose, ischemia can be provoked by the inflation of a balloon-tipped catheter within the coronary artery ${ }^{28}$ or by the external ligature of this artery. ${ }^{47}$ This creates an ideal substrate for arrhythmias by causing heterogeneities in terms of excitability, refractory period and conduction. ${ }^{46}$ The inconvenient is that it is difficult to predict the time and rate of $\mathrm{VF}$ occurrence. ${ }^{48-50}$

\section{Models of non-shockable cardiac arrest}

This situation is usually encountered in rodent models as nonshockable cardiac arrest could be easily induced through asphyxia or potassium chloride administration, which provokes pulseless electrical activity and primitive asystole, respectively. The clinical relevance of such cardiac arrest could not be generalized to other situations due to their low proportion as compared to shockable cardiac arrest in the clinical setting.

Asphyxial cardiac arrest: Asphyxia can easily be induced in laboratory animals by interrupting conventional mechanical ventilation in deeply anesthetized and paralyzed animals. ${ }^{27,29}$ After the onset of asphyxia, a brief increase in blood pressure is transiently observed before a lowflow period occurs with hypotension, bradycardia and progressive loss of arterial pulsatility, i.e., mean arterial pressure under $10-20 \mathrm{mmHg}$. Asphyxial cardiac arrest therefore includes an initial "low-flow period" prior to the actual "no-flow" with pulseless electrical activity (PEA) and then asystole. The time to loss of detectable arterial pulsation varies from 3-4minutes in rats, ${ }^{51} 5$ minutes in rabbits ${ }^{52,53}$ and 6 minutes in swine, ${ }^{33}$ respectively. The low-flow period leads to a progressive hypercarbia and transition to anaerobic metabolism, with combined respiratory and metabolic acidosis. Peripheral vasoconstriction also occurs before cardiac output deteriorates and blood circulation ceases. This "low-flow" phase could explain the worse prognosis in asphyxial 
cardiac arrest as compared to shockable cardiac arrest. As example, myocardial dysfunction is aggravated in pigs with asphyxial cardiac arrest as compared to VF with a significant decreased ejection fraction ( $31 \pm 1 \%$ vs $45 \pm 4 \%$, respectively). ${ }^{34}$ In another study, cerebral lesions were shown to be more severe in dogs submitted to asphyxia vs VF. ${ }^{33}$ However, the total duration of insult is typically longer in animal models of asphyxial cardiac arrest since it includes the initial lowflow period.

Asystole through potassium chloride administration: The intravenous administration of potassium chloride was also shown to induce a very reproducible cardiac arrest in rodents. ${ }^{9,13,14}$ It leads to a quasi-immediate cardiac arrest with asystole. Using appropriate dosage of potassium chloride (e.g., $0.08 \mathrm{mg} / \mathrm{g}$ in mice), ROSC can be easily obtained after the onset of CPR.

\section{Models of spontaneous cardiac arrest}

Animal models presenting high risk of cardiac arrest and VF are well described in the literature. Many models were developed in rodents through genetic modification, for the identification of risk factors and mechanistic studies. For example, it has been shown that the deletion of amino-acid residues in the gene encoding for the SCN5A cardiac sodium channel caused prolongation of repolarization and development of fatal ventricular arrhythmias in mice. ${ }^{54}$ In the same way, knock-out mice for cardiac connexin-43 died prematurely from sustained ventricular tachyarrhythmias, with a mean lifespan of $43.9 \pm 2.4$ days. ${ }^{55}$ In rabbits, the cardiac expression of KCNQ1 and $\mathrm{KCNH} 2$ human genes also induced a long QT syndrome, ${ }^{56}$ increasing the chance of ventricular tachycardia.

Spontaneous cardiac arrest can also occur in large animals in breeds presenting inherited arrhythmias. ${ }^{57}$ For example, some families of German shepherd dog can present ventricular tachycardia evolving into $\mathrm{VF}^{57}$ Boxer also can develop arrhythmogenic right ventricular dysplasia, characterized by sudden VF during exercise or excitement. ${ }^{25}$ Beyond genetic manipulation or selection of animals with inherited arrhythmias, increased hazard of spontaneous cardiac arrest can also be induced in animals through environmental modification and exposure to comorbidities. For example, rabbits exposed to hypercholesterolemic diet during 12weeks developed minimal coronary atherosclerosis and cardiac hypertrophy, but also prolonged QT interval and increased susceptibility to VF. ${ }^{58}$ However, such models present a lack of reproducibility and are typically used for the analysis of risk factors of VF. Their relevance for the evaluation of cardiac arrest consequences and CPR procedure is limited for obvious technical issues.

\section{Main end-points for cardiac arrest studies in animals}

\section{Neurological function and survival}

In many experimental studies, neurological outcomes and/or survival are used as primary end-points after cardiac arrest and CPR. Both parameters are very closely linked in laboratory animals as prolonged intensive care is rarely possible. Severe neurological sequels are therefore often associated with rapid mortality or early euthanasia for ethical reasons. In survivors, neurological function is typically assessed using clinical deficit scores with specific grids for each species. They are based on the clinical evaluation of consciousness, behavior, breathing, reflexes, and locomotion tests. ${ }^{9,11-13,28-31,41,50,53}$
Neurological recovery can be further assessed with quantitative behavioral tests in rodents, such as the T maze or the Morris maze test. ${ }^{59-61}$ In addition to the clinical evaluation of neurological function, histopathological damages are also usually evaluated in most experimental studies. ${ }^{62}$ End-points could be the number of ischemic neurons ${ }^{51}$ and the presence of other abnormalities such as edema or congestion of capillaries. ${ }^{33,63}$ Several circulating biomarkers of brain lesions could also be evaluated in animals as surrogate markers such as neuron specific enolase ${ }^{64}$ and protein $\mathrm{S} 100 .{ }^{65}$

\section{Hemodynamic evaluation}

Blood pressure is measured in most cardiac arrest studies in order to titrate vasopressors dosage after cardiac arrest. However, it is a poor marker of cardiac function which is typically assessed by echocardiography or left ventricle pressure measurement. Biomarkers can also be used to assess myocardial lesions, such as troponin I or brain natriuretic peptide plasma levels. ${ }^{66}$ Histological evaluations are required for the assessment of putative myocardial patchy infarction after cardiac arrest. ${ }^{34}$

\section{Multiorgan failure assessment and "sepsis-like" syn- drome}

Beyond neurological and myocardial responses, cardiac arrest also leads to multi-organ failure and renal, hepatic and mesenteric lesions. ${ }^{31}$ This leads to a "post-cardiac arrest" syndrome which also includes systemic inflammatory response, coagulopathy and adrenal insufficiency. ${ }^{67,68}$ In animal studies, this so-called "sepsislike syndrome" can be evidenced by the evaluation of the cytokines transcription levels ${ }^{53}$ and coagulation parameters, ${ }^{69}$ as well as numerous biomarkers for each particular organ. For example, adrenal insufficiency can be highlighted by comparing ACTH and cortisol concentrations,${ }^{70}$ while kidney failure could be evidenced by creatinine blood levels or urinary levels of $\mathrm{N}$ acetyl $\beta$ (D) glucosaminidase, $\gamma$ glutamyltransferase or $\beta 2$ microglobulin $^{71}$ failure could be evidenced by creatinine blood levels or urinary levels of $N$ acetyl $\beta(D)$ glucosaminidase, $\gamma$ glutamyltransferase or $\beta 2$ microglobulin. ${ }^{71,72}$

\section{Conclusion}

In conclusion, many animal models can be used for the investigation of cardiac arrest and cardiopulmonary resuscitation. This allows the evaluation of new devices for CPR (e.g., new debrillation devices or apparatus for automated massage) or new therapeutic approaches limiting the sequels of cardiac arrest after resuscitation (e.g., new approaches for hypothermia, new drugs, etc). The choice of the species and modality of induction of cardiac arrest is crucial for each particular study. It raises important questions for veterinary experimental research with possible applications in both human and veterinary medicine.

\section{Acknowledgements}

None.

\section{Conflict of interest and fundings}

All authors had equally contributed to the work. MB, MK and LZ declare that they have no competing of interest. RT is named as inventor on a patent application concerning cardiac arrest treatment (cooling with liquid ventilation [US 13/039415]). This topic is not described in the present review. 
This study was supported by Grant DBS20140930781 from the «Fondation pour la RechercheMedicale» (FRM).

\section{References}

1. Bougouin W, Lamhaut L, Marijon E, et al. Characteristics and prognosis of sudden cardiac death in Greater Paris: population-based approach from the Paris Sudden Death Expertise Center (Paris-SDEC). Intensive Care Med. 2014;40(6):846-854.

2. Dumas F, Cariou A. Epidemiology, prognostic data of cardiac arrest in 2014. Presse Médicale Paris Fr. 2014;43(7-8):768-774.

3. Monsieurs KG, Nolan JP, Bossaert LL, et al. European Resuscitation Council Guidelines for Resuscitation 2015: Section 1. Executive summary. Resuscitation. 2015;95:1-80.

4. Neumar RW, Shuster M, Callaway CW, et al. Part 1: Executive Summary: 2015 American Heart Association Guidelines Update for Cardiopulmonary Resuscitation and Emergency Cardiovascular Care. Circulation. 2015;132(18 Suppl 2):S315-S367.

5. Nichol G, Leroux B, Wang H, et al. Trial of Continuous or Interrupted Chest Compressions during CPR. N Engl J Med. 2015;373(23):2203-2214.

6. Ciszek B, Skubiszewska D, Ratajska A. The anatomy of the cardiac veins in mice. $J$ Anat. 2007;211(1):53-63.

7. Papadimitriou D, Xanthos T, Dontas I, et al. The use of mice and rats as animal models for cardiopulmonary resuscitation research. Lab Anim. 2008;42(3):265-276.

8. Song L, Weil MH, Tang $\mathrm{W}$, et al. Cardiopulmonary resuscitation in the mouse. J Appl Physiol. 2002;93(4):1222-1226.

9. Abella BS, Zhao D, Alvarado J, et al. Intra-arrest cooling improves outcomes in a murine cardiac arrest model. Circulation. 2004;109(22):2786-2791.

10. Hutchens MP, Kosaka Y, Zhang W, et al. Estrogen-mediated renoprotection following cardiac arrest and cardiopulmonary resuscitation is robust to GPR30 gene deletion. PloS One. 2014;9(6):e99910.

11. Kida K, Shirozu K, Yu B, et al. Beneficial effects of nitric oxide on outcomes after cardiac arrest and cardiopulmonary resuscitation in hypothermia-treated mice. Anesthesiology. 2014;120(4):880-889.

12. Li J, Wang H, Zhong Q, et al. A novel pharmacological strategy by PTEN inhibition for improving metabolic resuscitation and survival after mouse cardiac arrest. Am J Physiol Heart Circ Physiol. 2015;308(11):H1414-H1422.

13. Minamishima S, Kida K, Tokuda K, et al. Inhaled nitric oxide improves outcomes after successful cardiopulmonary resuscitation in mice. Circulation. 2011;124(15):1645-1653.

14. Zhao D, Abella BS, Beiser DG, et al. Intra-arrest cooling with delayed reperfusion yields higher survival than earlier normothermic resuscitation in a mouse model of cardiac arrest. Resuscitation. 2008;77(2):242-249.

15. Jung B, Odening KE, Dall'Armellina E, et al. A quantitative comparison of regional myocardial motion in mice, rabbits and humans using in-vivo phase contrast CMR. J Cardiovasc Magn Reson. 2012;14:87.

16. Nerbonne JM. Studying Cardiac Arrhythmias in the Mouse-A Reasonable Model for Probing Mechanisms? Trends Cardiovasc Med. 2004;14(3):83-93.

17. Traystman RJ. Animal Models of Focal and Global Cerebral Ischemia. ILAR J. 2003;44(2):85-95.

18. Vermeulen JT, Mcguire MA, Opthof T, et al. Triggered activity and automaticity in ventricular trabeculae of failing human and rabbit hearts. Cardiovasc Res. 1994;28(10):1547-1554.
19. Baker AJ, Zornow MH, Grafe MR, et al. Hypothermia prevents ischemia-induced increases in hippocampal glycine concentrations in rabbits. Stroke. 1991;22(5):666-673.

20. Brown A, Woods S, Skinner R, et al. Neurological assessment scores in rabbit embolic stroke models. Open Neurol J. 2013;7:38-43.

21. Barouxis D, Chalkias A, Syggelou A, et al. Research in human resuscitation: what we learn from animals. J Matern. Fetal Neonatal Med. 2012;25(Suppl 5):44-46.

22. Xanthos T, Bassiakou E, Koudouna E, et al. Baseline hemodynamics in anesthetized landrace-large white swine: reference values for research in cardiac arrest and cardiopulmonary resuscitation models. $J$ Am Assoc Lab Anim Sci. 2007;46(5):21-25.

23. Swindle MM, Makin A, Herron AJ, et al. Swine as models in biomedical research and toxicology testing. Vet Pathol. 2012;49(2):344-356.

24. Lee KW, Everett TH $4^{\text {th }}$, Rahmutula D, et al. Pirfenidone prevents the development of a vulnerable substrate for atrial fibrillation in a canine model of heart failure. Circulation. 2006;114(16):1703-1712.

25. Stengl M. Experimental models of spontaneous ventricular arrhythmias and of sudden cardiac death. Physiol Res Acad Sci Bohemoslov. 2010;59 Suppl 1:S25-S31.

26. Bircher N, Safar P. Cerebral preservation during cardiopulmonary resuscitation. Crit Care Med. 1985;13(3):185-190.

27. Fries M, Brücken A, Çizen A, et al. Combining xenon and mild therapeutic hypothermia preserves neurological function after prolonged cardiac arrest in pigs. Crit Care Med. 2012;40(4):1297-1303.

28. Gong P, Hua R, Zhang Y, et al. Hypothermia-induced neuroprotection is associated with reduced mitochondrial membrane permeability in a swine model of cardiac arrest. J Cereb Blood Flow Metab. 2013;33(6):928-934.

29. Guan J, Barbut D, Wang H, et al. A comparison between head cooling begun during cardiopulmonary resuscitation and surface cooling after resuscitation in a pig model of cardiac arrest. Crit Care Med. 2008;36(11 Suppl):S428-S433.

30. Sideris G, Magkoutis N, Sharma A, et al. Early coronary revascularization improves $24 \mathrm{~h}$ survival and neurological function after ischemic cardiac arrest. A randomized animal study. Resuscitation. 2014;85(2):292-298.

31. Yannopoulos D, Segal N, Matsuura T, et al. Ischemic post-conditioning and vasodilator therapy during standard cardiopulmonary resuscitation to reduce cardiac and brain injury after prolonged untreated ventricular fibrillation. Resuscitation. 2013;84(8):1143-1149.

32. Kamohara T, Weil MH, Tang W, et al. A Comparison of Myocardial Function after Primary Cardiac and Primary Asphyxial Cardiac Arrest. Am J Respir Crit Care Med. 2001;164(7):1221-1224.

33. Vaagenes P, Safar P, Moossy J, et al. Asphyxiation versus ventricular fibrillation cardiac arrest in dogs.: Differences in cerebral resuscitation effects - a preliminary study. Resuscitation. 1997;35(1):41-52.

34. Wu CJ, Li CS, Zhang Y, et al. Differences of postresuscitation myocardial dysfunction in ventricular fibrillation versus asphyxiation. Am J Emerg Med. 2013;31(12):1690-1696.

35. Karagueuzian HS, Chen P-S. Cellular mechanism of reentry induced by a strong electrical stimulus Implications for fibrillation and defibrillation. Cardiovasc Res. 2001;50(2):251-262.

36. Ayoub IM, Kolarova J, Gazmuri RJ. Cariporide given during resuscitation promotes return of electrically stable and mechanically competent cardiac activity. Resuscitation. 2010;81(1):106-110. 
37. Huang L, Weil MH, Tang W, et al. Comparison between dobutamine and levosimendan for management of postresuscitation myocardial dysfunction. Crit Care Med. 2005;33(3):487-491.

38. Chen MH, Liu TW, Xie L, et al. A simpler cardiac arrest model in the mouse. Resuscitation. 2007;75(2):372-379.

39. Chen MH, Liu TW, Xie L, et al. A simpler cardiac arrest model in rats. Am J Emerg Med. 2007;25(6):623-630.

40. Chenoune M, Lidouren F, Adam C, et al. Ultrafast and Whole-Body Cooling With Total Liquid Ventilation Induces Favorable Neurological and Cardiac Outcomes After Cardiac Arrest in Rabbits. Circulation. 2011;124(8):901-911

41. Darbera L, Chenoune M, Lidouren F, et al. Hypothermic Liquid Ventilation Prevents Early Hemodynamic Dysfunction and Cardiovascular Mortality After Coronary Artery Occlusion Complicated by Cardiac Arrest in Rabbits. Crit Care Med. 2013;41(12):e457-e465.

42. Hutin A, Lidouren F, Kohlhauer M, et al. Total liquid ventilation offers ultra-fast and whole-body cooling in large animals in physiological conditions and during cardiac arrest. Resuscitation. 2015;93:69-73.

43. Huang L, Weil MH, Sun S, et al. Levosimendan improves postresuscitation outcomes in a rat model of CPR. J Lab Clin Med. 2005;146(5):256-261.

44. Lin J, Xiao-xing L, Li H, et al. Model of cardiac arrest in rats by transcutaneous electrical epicardium stimulation. Resuscitation. 2010;81(9):1197-1204.

45. Chen MH, Liu TW, Xie L, et al. Ventricular fibrillation induced by transoesophageal cardiac pacing: A new model of cardiac arrest in rats. Resuscitation. 2007;74(3):546-551.

46. Di Diego JM, Antzelevitch C. Ischemic ventricular arrhythmias: Experimental models and their clinical relevance. Heart Rhythm. 2011;8(12):1963-1968.

47. Tissier R, Hamanaka K, Kuno A, et al. Total liquid ventilation provides ultra-fast cardioprotective cooling. $\mathrm{J} \mathrm{Am}$ Coll Cardiol. 2007;49(5):601-605.

48. Cohen MV, Yang XM, Downey JM. Conscious rabbits become tolerant to multiple episodes of ischemic preconditioning. Circ Res. 1994;74(5):998-1004.

49. Opitz CF, Mitchell GF, Pfeffer MA, et al. Arrhythmias and death after coronary artery occlusion in the rat. Continuous telemetric ECG monitoring in conscious, untethered rats. Circulation. 1995;92(2):253-261.

50. Ristagno G, Fumagalli F, Russo I, et al. Postresuscitation Treatment With Argon Improves Early Neurological Recovery in a Porcine Model of Cardiac Arrest. Shock. 2014;41(1):72-78.

51. Katz L, Ebmeyer U, Safar P, et al. Outcome Model of Asphyxial Cardiac Arrest in Rats. J Cereb Blood Flow Metab. 1995;15(6):1032-1039.

52. Cour M, Loufouat J, Paillard M, et al. Inhibition of mitochondrial permeability transition to prevent the post-cardiac arrest syndrome: a pre-clinical study. Eur Heart J. 2011;32(2):226-235.

53. Kohlhauer M, Lidouren F, Remy-Jouet I, et al. Hypothermic Total Liquid Ventilation Is Highly Protective Through Cerebral Hemodynamic Preservation and Sepsis-Like Mitigation After Asphyxial Cardiac Arrest. Crit Care Med. 2015;43(10):e420-e430.

54. Nuyens D, Stengl M, Dugarmaa S, et al. Abrupt rate accelerations or premature beats cause life-threatening arrhythmias in mice with long-QT3 syndrome. Nat Med. 2001;7(9):1021-1027.
55. Danik SB, Liu F, Zhang J, et al. Modulation of cardiac gap junction expression and arrhythmic susceptibility. Circ Res. 2004;95(10):1035-1041.

56. Brunner M, Peng X, Liu GX, et al. Mechanisms of cardiac arrhythmias and sudden death in transgenic rabbits with long QT syndrome. J Clin Invest. 2008;118(6):2246-2259.

57. Moïse NS. Inherited arrhythmias in the dog. Cardiovasc Res. 1999;44(1):37-46

58. Liu YB, Wu CC, Lu LS, et al. Sympathetic nerve sprouting, electrical remodeling, and increased vulnerability to ventricular fibrillation in hypercholesterolemic rabbits. Circ Res. 2003;92(10):1145-1152.

59. Hickey RW, Akino M, Strausbaugh S, et al. Use of the Morris water maze and acoustic startle chamber to evaluate neurologic injury after asphyxial arrest in rats. Pediatr Res. 1996;39(1):77-84

60. Schreckinger M, Geocadin RG, Savonenko A, et al. Long-lasting cognitive injury in rats with apparent full gross neurological recovery after short-term cardiac arrest. Resuscitation. 2007;75(1):105-113.

61. Undén J, Sjölund C, Länsberg J-K, et al. Post-ischemic continuous infusion of erythropoeitin enhances recovery of lost memory function after global cerebral ischemia in the rat. BMC Neurosci. 2013;14:27.

62. Radovsky A, Safar P, Sterz F, et al. Regional prevalence and distribution of ischemic neurons in dog brains 96 hours after cardiac arrest of 0 to 20minutes. Stroke. 1995;26(11):2127-2133.

63. Varvarousi G, Johnson EO, Goulas S, et al. Combination pharmacotherapy improves neurological outcome after asphyxial cardiac arrest. $R e$ suscitation. 2012;83(4):527-532.

64. Zhao H, Li CS, Gong P, et al. Molecular mechanisms of therapeutic hypothermia on neurological function in a swine model of cardiopulmonary resuscitation. Resuscitation. 2012;83(7):913-920.

65. Pokela M, Anttila V, Rimpiläinen J, et al. Serum S-100beta protein predicts brain injury after hypothermic circulatory arrest in pigs. Scand Cardiovasc J. 2000;34(6):570-574.

66. Lu X, Ma L, Sun S, et al. The effects of the rate of postresuscitation rewarming following hypothermia on outcomes of cardiopulmonary resuscitation in a rat model. Crit Care Med. 2014;42(2):e106-e113.

67. Adrie C, Laurent I, Monchi M, et al. Postresuscitation disease after cardiac arrest: a sepsis-like syndrome? Curr Opin Crit Care. 2004;10(3):208-212.

68. Negovsky VA. The second step in resuscitation - the treatment of the 'post-resuscitation' disease. Resuscitation. 1972;1(1):1-7.

69. White NJ, Leong BS-H, Brueckner J, et al. Coagulopathy during cardiac arrest and resuscitation in a swine model of electrically induced ventricular fibrillation. Resuscitation. 2011;82(7):925-931.

70. Kornberger E, Prengel AW, Krismer A, et al. Vasopressin-mediated adrenocorticotropin release increases plasma cortisol concentrations during cardiopulmonary resuscitation. Crit Care Med. 2000;28(10):3517-3521.

71. Tissier R, Giraud S, Quellard N, et al. Kidney Protection by Hypothermic Total Liquid Ventilation after Cardiac Arrest in Rabbits. Anesthesiology. 2014;120(4):861-869.

72. Debaty G, Metzger A, Rees J, et al. Enhanced perfusion during advanced life support improves survival with favorable neurologic function in a porcine model of refractory cardiac arrest. Crit Care Med. 2015;43(5):1087-1095. 